Document downloaded from:

http://hdl.handle.net/10251/39083

This paper must be cited as:

Adolfo Ballester-Bolinches; Cosme-Llópez, E.; Esteban Romero, R. (2013). Algorithms for permutability in finite groups. Central European Journal of Mathematics. 11(11):1914-1922. doi:10.2478/s11533-013-0299-4.

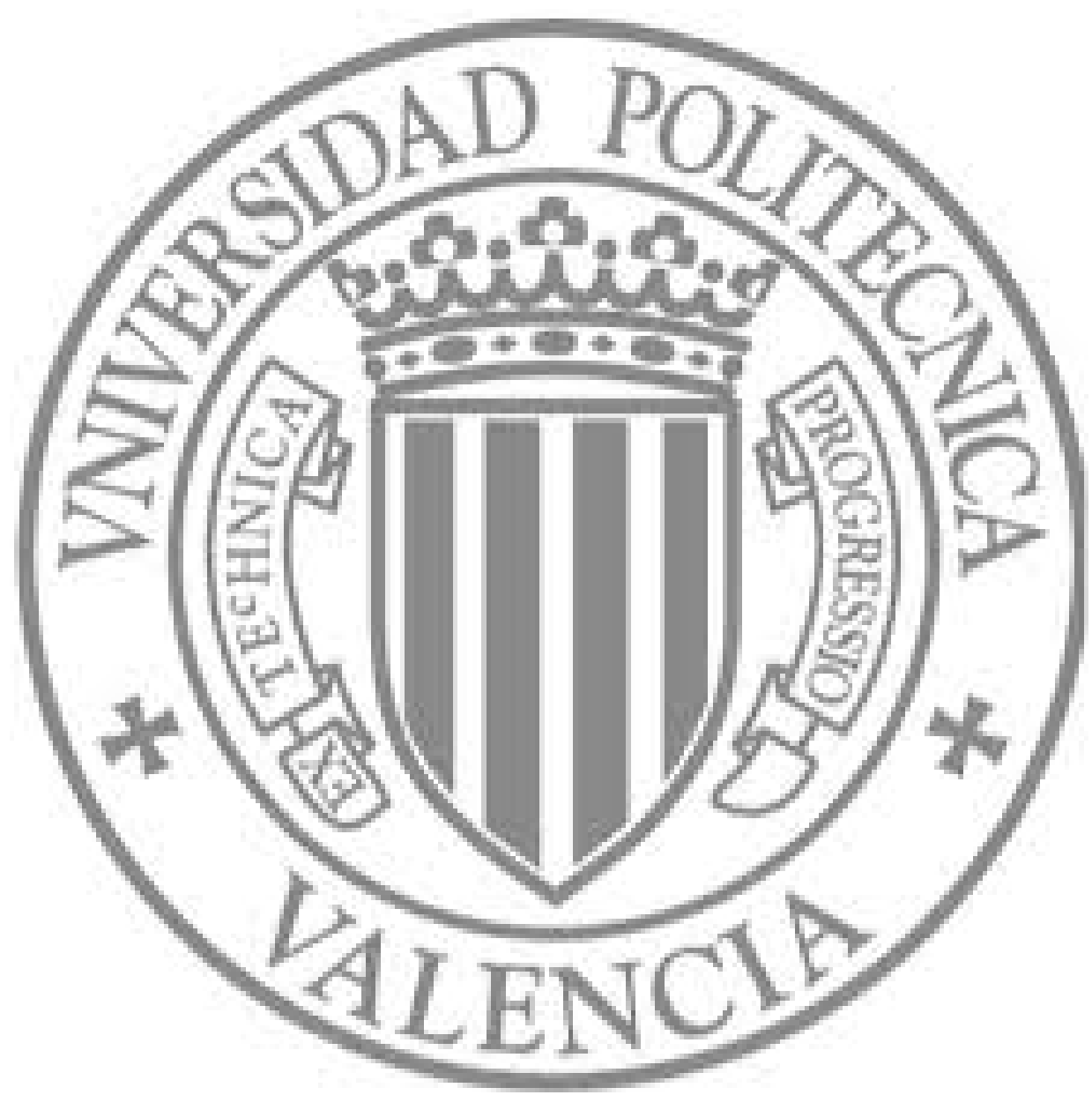

The final publication is available at

http://dx.doi.org/10.2478/s11533-013-0299-4

Copyright Springer Verlag (Germany) 
This paper has been published in Central European Journal of Mathematics, 11(11):1914-1922 (2013).

Copyright 2013 by Springer-Verlag and Versita Sp. z o.o.

The final publication is available at www.springerlink.com.

http://link.springer.com/article/10.2478/s11533-013-0299-4

http://dx.doi.org/10.2478/s11533-013-0299-4 


\title{
Algorithms for permutability in finite groups
}

\author{
A. Ballester-Bolinches* $\quad$ E. Cosme-Llópez ${ }^{\dagger}$ \\ R. Esteban-Romero ${ }^{\ddagger}$
}

31st August 2013

\begin{abstract}
In this paper we describe some algorithms to identify permutable and Sylow-permutable subgroups of finite groups, Dedekind and Iwasawa finite groups, and finite T-groups (groups in which normality is transitive), PT-groups (groups in which permutability is transitive), and PST-groups (groups in which Sylow permutability is transitive). These algorithms have been implemented in a package for the computer algebra system GAP.

Keywords: finite group, permutable subgroup, S-permutable subgroup, Dedekind group, Iwasawa group, T-group, PT-group, PSTgroup, algorithm
\end{abstract}

Mathematics Subject Classification (2010): 20D10, 20D20, 20-04

\section{Introduction}

In this paper we will only deal with finite groups. Therefore the word group will be understood as a synonym of finite group.

Many papers have recently dealt with the classes of groups in which normality, permutability, and Sylow permutability are transitive relations, the so-called T-groups, PT-groups, and PST-groups, respectively. Chapter 2 of

${ }^{*}$ Departament d'Àlgebra, Universitat de València; Dr. Moliner, 50; E-46100 Burjassot (València), Spain; email: Adolfo.Ballester@uv.es

†Departament d'Àlgebra, Universitat de València; Dr. Moliner, 50; E-46100 Burjassot (València), Spain; email: enriccosme@gmail.com

${ }^{\ddagger}$ Institut Universitari de Matemàtica Pura i Aplicada, Universitat Politècnica de València; Camí de Vera, s/n; E-46022 València, Spain; email: resteban@mat.upv.es. Current address: Departament d'Àlgebra, Universitat de València; Dr. Moliner, 50; E-46100 Burjassot (València), Spain; email: Ramon.Esteban@uv.es 
the book [6] contains an introduction to these classes of groups, as well as many other references about them. On the other hand, the current development of computational group theory has made of computational algebra systems like GAP [11] a powerful tool in the research in group theory.

In this paper we present some algorithms to identify permutable and S-permutable subgroups in finite groups, to find whether a group has all subgroups normal or permutable, and to check whether a soluble group is a T-group, a PT-group, and a PST-group. These algorithms have been implemented in a package for GAP [11] (see [4]).

\section{Permutability}

Recall that two subgroups $A$ and $B$ of a group $G$ permute when $A B=B A$ or, equivalently, when $A B$ is a subgroup of $G$. Recall also that a subgroup $H$ of a group $G$ is permutable (respectively, $S$-permutable in $G$ ) if it permutes with all subgroups (respectively, all Sylow subgroups) of $G$.

\subsection{A test to check whether two subgroups permute}

We can apply the following result in order to check whether two subgroups of a group permute.

Theorem 2.1 (see [6, Lemma 1.1.3]). Two subgroups $A$ and $B$ of a group $G$ permute if and only if

$$
|\langle A, B\rangle|=\frac{|A||B|}{|A \cap B|} .
$$

Remark 2.2. The efficiency of this test depends strongly on the implementation of good algorithms to compute the intersection of two subgroups of a group with a given representation.

\subsection{Dedekind groups}

Recall that a Dedekind group is a group with all subgroups normal. By [12, Chapter III, Satz 7.12], Dedekind groups are nilpotent and their Sylow $p$ groups for a prime $p$ are abelian or direct products of a quaternion group of order 8 and an elementary abelian 2-group (the latter only if $p=2$ ). The next result characterises non-abelian Dedekind 2-groups.

Theorem 2.3. A non-abelian 2-group $G$ is Dedekind if and only if the Frattini subgroup $\Phi(G)$ of $G$ has order 2 , the centre $\mathrm{Z}(G)$ of $G$ has exponent 2 and 
index 4, and the preimages of the generators of $G / \mathrm{Z}(G)$ under the natural epimorphism from $G$ onto $G / \mathrm{Z}(G)$ have order 4 .

Proof. By [12, Chapter III, Satz 7.12], a non-abelian Dedekind 2-group is isomorphic to a direct product of a quaternion group of order 8 and an elementary abelian 2-group. Hence these groups satisfy all conditions of the theorem.

Conversely, suppose that $G$ is a 2-group such that $\Phi(G)$ has order 2 and $\mathrm{Z}(G)$ has exponent 2 and index 4 . Note that $G / \mathrm{Z}(G)$ cannot be cyclic, since otherwise $G$ would be abelian. Therefore $G / \mathrm{Z}(G)$ is an elementary abelian group of order 4. Let $x \mathrm{Z}(G)$ and $y \mathrm{Z}(G)$ be non-trivial different elements of $G / \mathrm{Z}(G)$. By hypothesis, $x$ and $y$ have order 4 . Since in a 2-group $\Phi(G)=$ $G^{\prime} G^{2}$, where $G^{2}$ denotes the subgroup of $G$ generated by the squares of the elements of $G$, we conclude that $x^{2}=y^{2}=z$ and $[x, y] \in\langle z\rangle$, where $\Phi(G)=\langle z\rangle$. Assume now that $[x, y]=1$. Since $G=\langle Z(G), x, y\rangle$, we conclude that $x \in \mathrm{Z}(G)$, contrary to the choice of $x$. It follows that $[x, y]=z$. Let $Q=\langle x, y\rangle$. Then $x^{y}=x[x, y]=x z=x^{3}$ and so $Q$ is isomorphic to the quaternion group of order 8 . Let $E$ be a complement of $\langle z\rangle$ in the elementary abelian group $\mathrm{Z}(G)$. Since $Q \cap \mathrm{Z}(G)=\langle z\rangle$, it follows that $Q \cap E=1$. Moreover, $[Q, E]=1$. Hence $G=Q \times E$ is a non-abelian Dedekind group by [12, Chapter III, Satz 7.12]. This completes the proof of the theorem.

\subsection{Iwasawa groups}

An Iwasawa group is a group with all subgroups permutable. It is clear that Iwasawa groups are nilpotent. In fact, they coincide with the nilpotent groups whose subgroup lattice is modular. A nilpotent group is Iwasawa if and only if its Sylow $p$-subgroups for all primes $p$ are Iwasawa. The following result (see [16, Theorem 2.3.1]) is basic to identify whether a $p$-group is Iwasawa.

Theorem 2.4. A p-group $G$ has modular subgroup lattice if and only if

- $G$ is a direct product of a quaternion group $Q_{8}$ of order 8 with an elementary abelian 2-group, or

- $G$ contains an abelian normal subgroup $A$ with cyclic factor group $G / A$; further there exists an element $b \in G$ with $G=A\langle b\rangle$ and a positive integer $s$ such that $b^{-1} a b=a^{1+p^{s}}$ for all $a \in A$, with $s \geq 2$ in case $p=2$

As in [16, page 60], we say that a triple $(A, b, s)$ is an Iwasawa triple if $A$ is an abelian normal subgroup of $G, b \in G$, and $s$ is a positive integer which is at least 2 in case $p=2$ such that $G=A\langle b\rangle$ and $b^{-1} a b=a^{1+p^{s}}$ for all $a \in A$. 
In order to find an Iwasawa triple for an Iwasawa group $G$, we define a function that computes an Iwasawa triple in which a given normal abelian subgroup $A$ appears, if this triple exists. This is done in the following way:

\section{Algorithm IwasawaTripleWithSubgroup}

Input: a $p$-group $G$ and a normal abelian subgroup $A$ such that $G / A$ is cyclic

Output: an Iwasawa triple $(A, b, s)$ of $G$ containing $A$ or fail if it there is no such triple

\section{Method:}

- Check that each generator $a_{i}$ of $A=\left\langle a_{1}, \ldots, a_{m}\right\rangle$ generates a normal subgroup $\left\langle a_{i}\right\rangle$ of $G$. If this does not happen, return fail.

- Take a representative $b$ of a generator $b A$ of $G / A=\langle b A\rangle$.

- Take an element $a$ of $A$ whose order is the exponent of $A$. Note that $s \leq r=\log _{p}(\mathrm{o}(a))$.

- For each generator $c$ of $\langle b\rangle$ :

- Define $s$ to be the first $t$ between 1 and $r$ such that $a^{c}=a^{1+p^{t}}$ (if it exists).

- If this value exists, check whether $a_{i}^{c}=a_{i}^{1+p^{s}}$ for all $i \in$ $\{1,2, \ldots, m\}$. If this happens, return the triple $(A, c, s)$.

- If in the previous step we do not obtain any Iwasawa triple, return fail.

Now we are in a position to present the algorithm used to search for an Iwasawa triple in a $p$-group $G$. The main part of the algorithm consists of identifying the normal subgroups $N$ of $G$ such that $G / N$ is cyclic. In this case, there exists a series of normal subgroups $N=N_{r} \unlhd N_{r-1} \unlhd \cdots \unlhd N_{0}=G$ such that $\left|N_{i-1} / N_{i}\right|=p$ and $G / N_{i}$ is cyclic for $1 \leq i \leq r$. Therefore these subgroups will be found as a part of a chain of subgroups beginning with $G$, each maximal in the previous one and such that the smallest subgroup is normal and the quotient is cyclic. This search will be performed by means of a "depth first search" style algorithm. Now this computation can be very expensive in groups with large Frattini quotient, because the number of maximal subgroups is very large.

\section{Algorithm IwasawaTriple}


Input: a $p$-group $P$

Output: an Iwasawa triple $(A, b, s)$ of $G$ if there exists one, an empty list [] if the group is non-abelian Dedekind (for $p=2$ ), or fail if there is no such triple

\section{Method:}

- If $P$ is abelian, return $\left(P, 1, \log _{p} \exp P\right)$.

- If $P$ is not metabelian, return fail.

- If $p=2$ and $\exp P=4$, if $P$ is Dedekind, return an empty list [] (the group is Iwasawa, but no Iwasawa triple can exist), else return fail.

- Otherwise:

- Let $l=\{P\}$.

- Repeat (until the list $l$ is empty)

* Let $X$ be the last element of the list $l$.

* Delete $X$ from $l$.

* Compute the list $l_{1}$ of all maximal subgroups $T$ of $X$ such that $T$ is normal in $P$ and $P / T$ is cyclic.

* Append to $l$ the list $l_{1}$.

$*$ Select the list $l_{2}$ composed of the abelian groups in $l_{1}$.

* For each $X$ in $l_{2}$, look for an Iwasawa triple with $X$ in it (if it exists). If there exists one, return this value.

- Return fail.

Iwasawa $p$-groups are then identified as the groups in which this last function returns a value different from fail. This value can be either the empty list (for the case of non-abelian Dedekind 2-groups) or an Iwasawa triple. Before applying this algorithm, it might be convenient to compute a few pairs of cyclic subgroups of the $p$-group and to check that they permute, because otherwise the $p$-group cannot be Iwasawa. Note that in nilpotent groups with a nice computer representation this checking is not very expensive in general.

A normal subgroup with cyclic quotient can only appear as a "descendant" subgroup of a unique series of maximal subgroups, because the cyclic $p$-groups have a unique chief series. Therefore there can be no repetitions in the list $l$. The sequence followed by the algorithm ensures that the orders of the normal subgroups with cyclic quotient are decreasing while possible before taking another such subgroup of bigger order. 


\subsection{S-permutable subgroups}

In order to give an algorithm which allows us to decide whether or not a subgroup $H$ of a group $G$ is S-permutable in $G$, we use the following fact proved by Schmid [15] (see also [6, Proposition 1.2.18]).

Theorem 2.5. The subgroup $H$ of a group $G$ is $S$-permutable in $G$ if and only if for each prime $p$ there exists a Sylow p-subgroup $H_{p} / H_{G}$ of $H / H_{G}$ such that $\mathrm{O}^{p}(G) \leq \mathrm{N}_{G}\left(H_{p}\right)$.

Here the computation of the core can be expensive because the usual algorithms involve the calculation of intersections of subgroups. The computation of the quotient group can also be expensive, especially in insoluble groups.

The following result of Deskins and Kegel (see [6, Theorem 1.2.14]) gives a necessary condition for S-permutability and can be applied before in order to show that some subgroups are not S-permutable.

Theorem 2.6. If $H$ is an S-permutable subgroup of $G$, then $H / H_{G}$ is a nilpotent subnormal subgroup of $G / H_{G}$.

Algorithm IsSPermutable

Input: a group $G$ and a subgroup $H$ of $G$

Output: true if $H$ is S-permutable in $G$, false otherwise

\section{Method:}

- If $H$ is normal in $G$, return true.

- If $H$ is known to be permutable in $G$, return true.

- If $H / H_{G}$ is not nilpotent, return false.

- Let $\pi=\pi\left(H / H_{G}\right)$. For each $p \in \pi$ :

- Compute a Sylow $p$-subgroup $H_{p}$ of $H$.

- Compute the $p$-residual $\mathrm{O}^{p}(G)$.

- If $\left\langle H_{p}^{\mathrm{O}^{p}(G)}\right\rangle \not \leq H_{p} H_{G}$, return false.

- Return true.

In this context, the expression " $H$ is known to be permutable in $G$ " means that we have previously computed whether $H$ is permutable in $G$, that this information is stored, and that we know that the answer to this question is affirmative. If this information is not available, this test is skipped. In this way we take advantage of the mechanism of attributes and properties of GAP ([11]). Note that this algorithm does not require checking whether two subgroups permute (Theorem 2.1). 


\subsection{Permutable subgroups}

Our next goal is to give an algorithm to determine whether a subgroup $H$ of a group $G$ is permutable.

Theorem 2.7 (see [6, Theorem 1.2.2]). If a subgroup $H$ of a group $G$ permutes with the subgroups $X$ and $Y$ of $G$, then it also permutes with their join $\langle X, Y\rangle$.

It follows that $H$ is permutable in $G$ if and only if $H$ permutes with all cyclic subgroups of prime power order of $G$. However, the number of such cyclic subgroups in a given group can be very large and this can make such a test unsuitable. Moreover, as stated in Remark 2.2, checking that two subgroups permute can be an expensive operation because of the computation of intersections, especially when many such tests are required. Therefore we need some arguments in order to reduce, at least in some cases, the number of these tests.

First of all, the fact that permutable subgroups are S-permutable and subnormal can help us to show that some subgroups are not permutable. The fact that normal subgroups are permutable can be also used to give an affirmative answer in the case of normal subgroups.

The following result of Maier and Schmid [13] (see also [6, Corollary 1.5.6]) gives an interesting necessary condition for permutability.

Theorem 2.8. If $H$ is a permutable subgroup of a group $G$, then $H / H_{G} \leq$ $\mathrm{Z}_{\infty}\left(G / H_{G}\right)$.

Here, for a group $K, \mathrm{Z}_{\infty}(K)$ denotes the hypercentre of $K$, that is, the limit of the upper central series of $K$. The test for permutability is based on the following result.

Theorem 2.9. Let $G$ be a group, $H$ be a subgroup of $G$, and for every prime $p$ let $H_{p}$ be a Sylow p-subgroup of $H$. Then $H$ is permutable in $G$ if and only if $H / H_{G}$ is nilpotent and for every prime $p$ dividing $\left|H: H_{G}\right|, \mathrm{O}^{p}\left(G / H_{G}\right)$ centralises $H_{p} H_{G} / H_{G}$ and $H_{p} H_{G} / H_{G}$ permutes with all cyclic subgroups of all Sylow p-subgroups of $G / H_{G}$.

Proof. Suppose that $H$ is permutable in $G$. Then $H / H_{G}$ is permutable in $G / H_{G}$ by [6, Lemma 1.2.7]. By Theorem 2.8, $H / H_{G} \leq \mathrm{Z}_{\infty}\left(G / H_{G}\right)$. Since $H_{p} H_{G} / H_{G}$ is a Sylow $p$-subgroup of $H / H_{G}, \mathrm{O}^{p}\left(G / H_{G}\right) \leq \mathrm{C}_{G}\left(H_{p} H_{G} / H_{G}\right)$ by [6, Lemma 1.2.22]. Moreover $H / H_{G}$ permutes with all cyclic subgroups of $G / H_{G}$. Let $x H_{G}$ be an element of $p$-power order of $G / H_{G}$. Then $H / H_{G}$ permutes with $\left\langle x H_{G}\right\rangle$. By [6, Theorem 1.1.19] and the fact that $H / H_{G}$ is 
nilpotent, there exists a Sylow $p$-subgroup $Z_{p} / H_{G}$ of $\left(H / H_{G}\right)\left\langle x H_{G}\right\rangle$ such that $Z_{p} / H_{G}=\left(H_{p} H_{G} / H_{G}\right)\left\langle x H_{G}\right\rangle$. Thus $H_{p} H_{G} / H_{G}$ permutes with $\left\langle x H_{G}\right\rangle$.

Conversely, suppose that $H / H_{G}$ is nilpotent and that for all primes $p$ dividing $\left|H: H_{G}\right|, \mathrm{O}^{p}(G)$ normalises $H_{p} H_{G}$ and $H_{p} H_{G} / H_{G}$ permutes with all cyclic subgroups of all Sylow $p$-subgroups of $G / H_{G}$. It is enough to check that all cyclic subgroups of prime power order of $G$ permute with $H$. Let $C=\langle x\rangle$ be a cyclic subgroup of $p$-power order of $G$. Let $q$ be a prime dividing $\left|H: H_{G}\right|$ different from $p$. Since $\mathrm{O}^{q}\left(G / H_{G}\right)=\mathrm{O}^{q}(G) H_{G} / H_{G}$ centralises $H_{q} H_{G} / H_{G}, C H_{G} / H_{G}$ permutes with $H_{q} H_{G} / H_{G}$. By hypothesis, $C H_{G} / H_{G}$ permutes with $H_{p} H_{G} / H_{G}$. It follows that $C H_{G} / H_{G}$ permutes with $H / H_{G}$. In particular, $C$ permutes with $H$, as desired.

The last part of the algorithm (checking that the Sylow $p$-subgroup of $H / H_{G}$ permutes with all cyclic subgroups of $p$-power order of $G / H_{G}$ ) can be speeded up in the case $G / H_{G}$ has an Iwasawa Sylow $p$-subgroup. We consider that enforcing this test could help saving some time when there are many elements of $p$-power order in $G$, but there is no need to check permutability of all these subgroups. We also check whether the number of conjugate subgroups of the Sylow $p$-subgroup of $H / H_{G}$ is smaller than the number of conjugate subgroups of a Sylow $p$-subgroup of $G / H_{G}$. In this last case, it is enough to verify that all conjugates of the Sylow $p$-subgroup of $H / H_{G}$ permute with all cyclic subgroups of a given Sylow $p$-subgroup of $G$.

\section{Algorithm IsPermutable}

Input: a group $G$ and a subgroup $H$ of $G$

Output: true if $H$ is permutable in $G$, false otherwise

\section{Method:}

- If $H$ is normal in $G$, return true.

- If $H$ is known to be non-S-permutable in $G$, return false.

- If $G$ is nilpotent and known to be an Iwasawa group, return true.

- Repeat for MaxPermutTries times: Take a random element $u$ of $G$. If $H$ does not permute $\langle u\rangle$, then return false.

- If $H$ is not subnormal in $G$, return false.

- Compute $\bar{G}=G / H_{G}$ and $\bar{H}=H / H_{G}$.

- If $\bar{H}$ is not nilpotent, return false.

- Let $\pi=\pi\left(H / H_{G}\right)$. For each $p \in \pi$ : 
- Compute a Sylow $p$-subgroup $\bar{H}_{p}$ of $\bar{H}$.

- Compute the $p$-residual $\mathrm{O}^{p}(G)$.

- If $\mathrm{O}^{p}(G)$ does not centralise $\bar{H}_{p}$, return false.

- Compute a Sylow $p$-subgroup $\bar{G}_{p}$ of $G$.

- If $\bar{G}_{p}$ is not known to be Iwasawa, check whether $\bar{H}_{p}$ permutes with all cyclic subgroups of $p$-power order of $G$ or whether all conjugates of $\bar{H}_{p}$ permute with all cyclic subgroups of $G_{p}$, whatever needs less calculations. If any of this checks gives a false result, return false.

- Return true.

Once more, the computations of the core and the quotient can be expensive operations because they involve the calculation of intersections of subgroups. This is especially relevant in the case of insoluble groups. The algorithm also relies on effective algorithms for the identification of a few random elements of the group. The value of MaxPermutTries has been set by default to 10 .

\section{T-groups, PT-groups, and PST-groups}

Groups in which normality (respectively, permutability, Sylow permutability) is transitive are called T-groups (respectively, PT-groups, PST-groups). These classes have been widely studied, especially in the soluble universe (see, for instance, [6, Chapter 2]). We will present some algorithms to check whether a group is a T-group, a PT-group, or a PST-group.

For soluble groups, we will use a local approach. As in Robinson [14], we say that a group $G$ satisfies $\mathcal{C}_{p}$ whenever if $T$ is a subgroup of a Sylow $p$-subgroup $P$ of $G$, then $T$ is normal in $\mathrm{N}_{G}(P)$. According to Beidleman, Brewster, and Robinson [9], we say that a group $G$ satisfies $\mathcal{X}_{p}$ if every subgroup of a Sylow $p$-subgroup $P$ of $G$ is permutable in the corresponding Sylow normaliser $\mathrm{N}_{G}(P)$. Finally, according to Ballester-Bolinches and Esteban-Romero [5], we say that a group $G$ satisfies $\mathcal{Y}_{p}$ if whenever $H \leq K$ are two $p$-subgroups of $G$, then $H$ is S-permutable in $\mathrm{N}_{G}(K)$.

\subsection{Soluble PST-groups}

We begin with the identification of soluble PST-groups. We will use the following results of Ballester-Bolinches and Esteban-Romero [5] (see [6, Theorems 2.2.9 and 2.2.13]): 
Theorem 3.1. A group $G$ is a soluble PST-group if and only if for all primes $p, G$ satisfies $\mathcal{Y}_{p}$.

Theorem 3.2. Let $p$ be a prime. A group $G$ satisfies $\mathcal{Y}_{p}$ if and only if $G$ is p-nilpotent or $G$ has abelian Sylow p-subgroups and $G$ satisfies $\mathcal{C}_{p}$.

The latter condition is equivalent to saying that the elements of $\mathrm{N}_{G}(P)$ induce power automorphisms in $P$. We will give an algorithm to check whether a group with abelian Sylow $p$-subgroups satisfies $\mathcal{C}_{p}$.

Note that if the Sylow $p$-subgroup $P$ of a group $G$ is abelian and $G$ satisfies $\mathcal{C}_{p}$, then the $p^{\prime}$-elements of $\mathrm{N}_{G}(P)$ act as power automorphisms on $P$. These automorphisms are universal by [6, Theorem 1.3.2]. Furthermore, the order of the group of automorphisms of a cyclic group of order $p^{n}$ is $p^{n-1}(p-1)$, and it is cyclic if $p \neq 2$ by [12, Chapter I, Satz 4.6 and Satz 13.19]. In particular, $\mathrm{N}_{G}(P) / \mathrm{C}_{G}(P)$ must be a cyclic group. If $p$ is the smallest prime dividing $|G|$, in particular, if $p=2$, then $G$ must be $p$-nilpotent by a result of BallesterBolinches and Esteban-Romero ([5], see also [6, Corollary 2.2.18]). Hence we can consider an element $b$ such that $\mathrm{C}_{G}(P)\langle b\rangle=\mathrm{N}_{G}(P)$, an element $a$ of a set of generators of $P$ of the largest possible order, and we determine an element $r$ such that $1 \leq r \leq \mathrm{o}(a)-1$ and $a^{b}=a^{r}$. Then we check whether for all generators $c$ of $P$ the relation $c^{b}=c^{r}$ holds. In algorithmic form:

\section{Algorithm IsAbCp}

Input: a group $G$ and a prime $p$

Output: true or false, according to whether $G$ has an abelian Sylow $p$ subgroup and $G$ satisfies the property $\mathcal{C}_{p}$

\section{Method:}

- If $p$ does not divide $|G|$, return true.

- Let $P$ be a Sylow $p$-subgroup of $G$.

- If $P$ is not abelian, return false.

- If $p$ is the smallest prime dividing $|G|$, return true if the group is $p$-nilpotent, and false otherwise.

- If for some of the generators $c$ of $P,\langle c\rangle$ is not normal in $\mathrm{N}_{G}(P)$, return false.

- Consider $\mathrm{N}_{G}(P) / \mathrm{C}_{G}(P)$. If this quotient is trivial, return true.

- If this quotient is not cyclic, return $\mathrm{false}$.

- Take an element $a$ of $P$ of the largest possible order. 
- Take an element $b$ of $\mathrm{N}_{G}(P)$ such that $\mathrm{C}_{G}(P)\langle b\rangle=\mathrm{N}_{G}(P)$.

- Compute a value $r$ such that $1 \leq r \leq \exp (P)-1$ and $a^{b}=a^{r}$.

- If for all generators $t$ of $P t^{b}=t^{r}$, return true, else return false.

In order to check whether a soluble group is a PST-group, we only test that for all primes $p$ dividing its order, $G$ is $p$-nilpotent or $G$ as an abelian Sylow $p$-subgroup and satisfies $\mathcal{C}_{p}$.

Note that the property $\mathcal{Y}_{p}$ can be also defined for insoluble groups. Since currently the methods to check $p$-nilpotency in GAP work only for soluble groups, we have introduced a method to check $p$-nilpotency in insoluble groups in which a chief series has been computed.

\subsection{Soluble PT-groups and T-groups}

Let $p$ be a prime. The following theorem (see, for instance, [6, Theorems 2.2.2 and 2.2.4]) gives a local characterisation of soluble T-groups and soluble PTgroups:

Theorem 3.3. A group $G$ is a soluble T-group (respectively, PT-group) if and only if $G$ satisfies $\mathcal{C}_{p}$ (respectively, $\mathcal{X}_{p}$ ) for all primes $p$.

Other interesting properties which characterise soluble T- and PT-groups are equivalent to these properties, at least in the $p$-soluble universe. Our functions to check whether a group satisfies $\mathcal{C}_{p}$ or $\mathcal{X}_{p}$ for a prime $p$ are based on the following result ([6, Theorems 2.2.4]):

Theorem 3.4. A group $G$ satisfies $\mathcal{X}_{p}$ (respectively, $\mathcal{C}_{p}$ ) if and only if $G$ satisfies $\mathcal{Y}_{p}$ and the Sylow p-subgroups of $G$ are Iwasawa (respectively, Dedekind)

Therefore we can use the algorithm to check whether a group has an abelian Sylow $p$-subgroup and satisfies $\mathcal{C}_{p}$ as well as the algorithms to check whether the Sylow $p$-subgroups are Dedekind or Iwasawa in order to determine whether a group satisfies $\mathcal{C}_{p}, \mathcal{X}_{p}$, or $\mathcal{Y}_{p}$.

\subsection{Insoluble PST-, PT-, and T-groups}

For insoluble groups, we first observe that PST-, PT-, and T-groups have all chief factors simple. The default method for T-groups searches all normal subgroups of normal subgroups and checks whether they are normal. For insoluble PST-groups, we check that normal subgroups of normal subgroups are 
S-permutable, since this characterises PST-groups as shown in [7, 8]. Nevertheless, this approach is not valid for insoluble PT-groups, as the example in [1] shows. In this case, we just check the permutability of all subnormal subgroups. Other possible methods could include some of the ideas present in $[2,3,10]$. However, we have not implemented these methods so far because they require the computation of the $p$-radicals of all possible quotients of insoluble groups, which could be a time-consuming task for groups of large order.

\section{Some computations}

The use of random elements in the algorithms to check the property $\mathcal{C}_{p}$ and that a group is Dedekind seems to reduce the time for the computation. For instance, the determination of all PT-groups of order up to 200 without this randomness has taken 660 seconds on a computer with an Intel ${ }^{\circledR}$ Core $^{\mathrm{TM}}$ i5 M430@2.27GHz CPU and 4 Gb of RAM, while randomness has decreased this time to only 167 seconds.

Let $N=\left\langle n_{1}, n_{2}, n_{3}, n_{4}\right\rangle$ be an elementary abelian group with $n_{i}$ of order $3^{i}, 1 \leq i \leq 4$. Let $\alpha$ be the power automorphism of $N$ defined as $n_{i}^{\alpha}=n_{i}$, $1 \leq i \leq 4$, and let $G_{1}=[N]\langle\alpha\rangle$ be the corresponding semidirect product, of order $3^{13}$ and Frattini subgroup of index $3^{5}$. The algorithm to check that this group is Iwasawa takes about 45 seconds. Another way of proving that this group is Iwasawa is by checking that all pairs of cyclic subgroups permute. However, this computation was interrupted by the authors after more than 24 hours without any result. The check that all subnormal subgroups of defect two are permutable does not seem very promising here, because of the large number of such subgroups. The corresponding computation was also interrupted after more than 24 hours.

Now consider the automorphism $\beta$ of the same group $N$ defined by $n_{1}^{\beta}=$ $n_{1}, n_{2}^{\beta}=n_{2}^{4}, n_{3}^{\beta}=n_{3}^{4}, n_{4}^{\beta}=n_{4}^{10}$. Let $G_{2}=[N]\langle\beta\rangle$ be the corresponding semidirect product. Checking that this group of order $3^{12}$ and with $G_{2} / \Phi\left(G_{2}\right)$ of order $3^{5}$ is not Iwasawa has required 93.5 seconds without the use of random elements. In this case, all abelian normal subgroups with cyclic factor group have had to be computed. With the help of pairs of random elements the time has been reduced to only 52 milliseconds. The search for a subnormal subgroup of defect two which is not permutable has taken 203 seconds. 


\section{Acknowledgement}

This work has been supported by the grant MTM2010-19938-C03-01 (Ministerio de Ciencia e Innovación, Spain). The first author has also been supported by a project of the National Natural Science Foundation of China (11271085). The third author has been supported by the predoctoral grant AP2010-2764 (Programa FPU, Ministerio de Educación, Spain).

\section{References}

[1] A. Ballester-Bolinches, J. C. Beidleman, J. Cossey, R. Esteban-Romero, M. F. Ragland, and J. Schmidt. Permutable subnormal subgroups of finite groups. Arch. Math. (Basel), 92:549-557, 2009.

[2] A. Ballester-Bolinches, J. C. Beidleman, and H. Heineken. Groups in which Sylow subgroups and subnormal subgroups permute. Illinois J. Math, 47(1-2):63-69, 2003.

[3] A. Ballester-Bolinches, J. C. Beidleman, and H. Heineken. A local approach to certain classes of finite groups. Comm. Algebra, 31(12):59315942, 2003.

[4] A. Ballester-Bolinches, E. Cosme-Llópez, and R. Esteban-Romero. Permut - A GAP4 package to deal with permutability, v. 0.03.

[5] A. Ballester-Bolinches and R. Esteban-Romero. Sylow permutable subnormal subgroups of finite groups. J. Algebra, 251(2):727-738, 2002.

[6] A. Ballester-Bolinches, R. Esteban-Romero, and M. Asaad. Products of finite groups, volume 53 of de Gruyter Expositions in Mathematics. Walter de Gruyter, Berlin, 2010.

[7] A. Ballester-Bolinches, R. Esteban-Romero, and M. Ragland. A note on finite $\mathcal{P S} \mathcal{S}$-groups. J. Group Theory, 10(2):205-210, 2007.

[8] A. Ballester-Bolinches, R. Esteban-Romero, and M. Ragland. Corrigendum: A note on finite $\mathcal{P S} \mathcal{S}$-groups. J. Group Theory, 12(6):961-963, 2009 .

[9] J. C. Beidleman, B. Brewster, and D. J. S. Robinson. Criteria for permutability to be transitive in finite groups. J. Algebra, 222(2):400-412, 1999. 
[10] J. C. Beidleman and H. Heineken. Finite soluble groups whose subnormal subgroups permute with certain classes of subgroups. J. Group Theory, 6(2):139-158, 2003.

[11] The GAP Group. GAP - Groups, Algorithms, and Programming, Version 4.5.7, 2012.

[12] B. Huppert. Endliche Gruppen I, volume 134 of Grund. Math. Wiss. Springer Verlag, Berlin, Heidelberg, New York, 1967.

[13] R. Maier and P. Schmid. The embedding of quasinormal subgroups in finite groups. Math. Z., 131:269-272, 1973.

[14] D. J. S. Robinson. A note on finite groups in which normality is transitive. Proc. Amer. Math. Soc., 19:933-937, 1968.

[15] P. Schmid. Subgroups permutable with all Sylow subgroups. J. Algebra, 207:285-293, 1998.

[16] R. Schmidt. Subgroup lattices of groups, volume 14 of De Gruyter Expositions in Mathematics. Walter de Gruyter, Berlin, 1994. 